\title{
A NONHOMOGENEOUS LINEAR DIFFERENTIAL SYSTEM WITH INTERFACE CONDITIONS
}

\author{
ROBERT NEFF BRYAN
}

1. Introduction. The problem discussed in this paper is a boundaryvalue interface-condition problem related to the operator $L(Y ; Q)$ $=Y^{\prime}-P Y-H^{\prime \prime} Q$. The coefficients $P, H$, and $Q$ are real-valued $n \times n$ matrix functions defined on a bounded interval $[a, b]$ such that $P$ is continuous, $H$ is of bounded variation, and $Q$ is constant. The differentiation takes place on the set $S_{h}=\left\{x \in[a, b] \mid H^{\prime}(x)\right.$ exists $\}$, and the superscript indicates the transpose.

For the case $H=0$, several sets of auxiliary conditions have been considered in conjunction with the operator $L$, including two-point boundary conditions (Birkhoff and Langer [1]), integral boundary conditions (Whyburn [9] and Cole [3]) and interface conditions (Stallard [8], Whyburn and Pignani [7], Zettl [10], and Conti [4]).

Krall considered the case in which $Q$ is the operator $C Y(a)+D Y(b)$ and imposed integral boundary conditions in [6]. He also considered a more general case in which $H^{t^{\prime}} Q$ is replaced by a linear combination of such terms and imposed integral boundary conditions and interface conditions at finitely many points in [5].

One of the usual results in the studies referred to above is the definition of an adjoint system which is not always in the same form as the original system. Another is the definition of a Green's function. Theorems on compatibility have been included in some. In this paper, similar results appear for the system

(1a) $L(Y ; Q)=0$ on $S_{h}$,

(1b) $Y-H^{t} Q$ is absolutely continuous on $[a, b]$,

(1c) $U(Y)=A Y(a)+B Y(b)+\int_{a}^{b} d K Y=0$,

(1d) $V(Y)=C Y(a)+D Y(b)=Q$,

in which $K$ is an $n \times n$ matrix whose elements are of bounded variation on $[a, b]$, and $A, B, C$, and $D$ are $n \times n$ matrix constants such that the $2 n \times 2 n$ matrix

$$
\left(\begin{array}{ll}
A & B \\
C & D
\end{array}\right)
$$

is nonsingular.

Note that condition (1b) imposes the interface conditions $Y(x+)$

Presented to the Society, January 25, 1969 under the title $A$ linear differential system with interface conditions; received by the editors September 23, 1968. 
$-Y(x-)=\left[H^{t}(x+)-H^{t}(x-)\right] Q$ at each $x$ for which $H$ is discontinuous; i.e., at possibly infinitely many points of the interval $[a, b]$. This is one of the features of this exposition. The other is that the adjoint system has the same form as system (1).

2. The family of adjoint systems. We denote the set $\left\{x \in[a, b] \mid K^{\prime}(x)\right.$ exists $\}$ by $S_{k}$ and let $A^{*}, B^{*}, C^{*}$, and $D^{*}$ denote the $n \times n$ matrix constants satisfying

$C^{* t} A+A^{{ }^{*} t} C=I$, the identity matrix, $C^{* t} B+A^{* t} D=0$, and $D^{* t} A$ $+B^{*_{t}} C=0, D^{*_{t}} B+B^{* t} D=-I$.

Definition. The one-parameter family of systems adjoint to system (1) is defined by

(2a) $L^{*}(Z ; R)=-Z^{\prime}-P^{t} Z+K^{t^{\prime}} R=0$ on $S_{k}$,

(2b) $Z-K^{t} R$ is absolutely continuous on $[a, b]$,

(2c) $U^{*}(Z)=A^{*} Z(a)+B^{*} Z(b)+\int_{a}^{b} d H Z=0$,

(2d) $V^{*}(Z)=C^{*} Z(a)+D^{*} Z(b)=R$,

in which $R$, the parameter, is an $n \times n$ constant matrix.

To justify this choice of form for the adjoint system we present the following results.

Green's Formula. If $Y$ satisfies (1b) and $Z$ satisfies (2b), then

$$
\int_{a}^{b}\left[Z^{t} L(Y ; Q)-L^{* t}(Z ; R) Y\right]=\left.Z^{t} Y\right|_{a} ^{b}-\left(\int_{a}^{b} d H Z\right)^{t} Q-R^{t} \int_{a}^{b} d K Y .
$$

The proof requires two integrations by parts.

Lemma 1. If $Y$ satisfies (1bd) and $Z$ satisfies (2bd), then

$$
\int_{a}^{b}\left[Z^{t} L(Y ; Q)-L^{* t}(Z ; R) Y\right]=-U^{* t}(Z) V(Y)-V^{* t}(Z) U(Y) .
$$

Proof. Combining Green's formula with (1d) and (2d) we have the left side equal to

$$
\begin{aligned}
&\left.Z^{t} Y\right|_{a} ^{b}-\left(\int_{a}^{b} d H Z\right)^{t} V(Y)-V^{* t}(Z) \int_{a}^{b} d K Y \\
&=\left.Z^{t} Y\right|_{a} ^{b}+\left[A^{*} Z(a)+B^{*} Z(b)\right]{ }^{t} V(Y)+V^{* t}(Z)[A Y(a)+B Y(b)] \\
&-U^{* t}(Z) V(Y)-V^{* t}(Z) U(Y) \\
&=-U^{* t}(Z) V(Y)-V^{* t}(Z) U(Y) .
\end{aligned}
$$

The justification for the last step is that our choice of $A^{*}, B^{*}, C^{*}$, and $D^{*}$ reduces the sum of the first three terms to 0 . See [2] for de- 
tails. As an immediate consequence we have the adjoint criterion theorem.

Theorem 1. If $Y$ satisfies (1bcd) and $Z$ satisfies (2bcd), then

$$
\int_{a}^{b}\left[Z^{t} L(Y ; Q)-L^{* t}(Z ; R) Y\right]=0 .
$$

3. Solution of the system. Let $\Phi$ denote the fundamental matrix of $Y^{\prime}=P Y$ which satisfies $Y(a)=I$. Denote $V(\Phi)$ by $\Delta$ and $V^{*}\left(\Phi^{-1 t}\right)$ by $\Delta^{*}$.

Lemma 2. If $\Delta$ is nonsingular, then system (1abd) has at most one solution for each $Q$.

Proof. Let $Y$ be the difference of two solutions, $Y_{1}$ and $Y_{2}$. Then $Y^{\prime}=P Y$ on $S_{h}$. Also $Y$ is a.c. on $[a, b]$. Hence $Y^{\prime}=P Y$ on $[a, b]$, so there exists a $C$ such that $Y=\Phi C$. But $V(Y)=V\left(Y_{1}\right)-V\left(Y_{2}\right)=Q-Q$ $=0=V(\Phi) C=\Delta C$. Hence, $C=0$ so that $Y=0$.

DeFinition. Let

$$
\begin{array}{rlrl}
G_{0}(x, t) & =\frac{1}{2} \Phi(x) \Phi^{-1}(t) & & \text { for } a \leqq t<x \leqq b \\
& =-\frac{1}{2} \Phi(x) \Phi^{-1}(t) & \text { for } a \leqq x<t \leqq b, \\
G_{0}^{*}(x, t) & =-G_{0}^{t}(t, x), & \\
J(x)=\int_{a}^{b} G_{0}(x, t) d H^{t}(t), \text { and } J^{*}(x)=\int_{a}^{b} G_{0}^{*}(x, t) d K^{t}(t) .
\end{array}
$$

We note that $J$ satisfies (1ab) with $Q=I$ and that $J^{*}$ satisfies (2ab) with $R=I$. Hence, any function of the form $Y=\Phi C+J Q$ satisfies (1ab). To find the $C$ required for $Y$ to satisfy (1d) we apply $V$ to $Y$ to obtain $V(Y)=V(\Phi) C+V(J) Q=\Delta C+V(J) Q=Q$. Assuming $\Delta^{-1}$ exists, we have $Y=\left\{\Phi \Delta^{-1}[I-V(J)]+J\right\} Q$. Using the notation $Y_{0}=\Phi \Delta^{-1}[I-V(J)]+J$, we can state the theorem that has just been proved.

THEOREM 2. If $\Delta$ is nonsingular, then $Y$ is a solution of (1abd) if and only if $Y=Y_{0} Q$.

Letting $Z_{0}=\Phi^{-1 t} \Delta^{*-1}\left[I-V^{*}\left(J^{*}\right)\right]+J^{*}$, we have the analogous theorem for adjoint systems.

THEOREM 3. If $\Delta^{*}$ is nonsingular, then $Z$ is a solution of (2abd) if and only if $Z=Z_{0} R$.

THEOREM 4. If $\Delta$ is nonsingular, then there exists a $Q \neq 0$ such that 
system (1) has a nontrivial solution if and only if $U\left(Y_{0}\right)$ is singular.

Proof. If $U\left(Y_{0}\right)$ is singular, then there is a $Q \neq 0$ such that $U\left(Y_{0}\right) Q=0 . Y=Y_{0} Q$ is the desired solution.

If $U\left(Y_{0}\right)$ is nonsingular and $Y$ is a nontrivial solution of (1), then $Y$ satisfies (1abd) so $Y=Y_{0} Q$ and $Q \neq 0$. But then $U(Y)=U\left(Y_{0}\right) Q$ $\neq 0$. This gives a contradiction, which completes the proof.

Theorem 4, applied to the adjoint system, gives us the following:

THEOREM 5. If $\Delta^{*}$ is nonsingular, then there exists an $R \neq 0$ such that system (2) has a nontrivial solution if and only if $U^{*}\left(Z_{0}\right)$ is singular.

As a preface to the theorem relating the compatibility of the two systems, we prove a lemma.

Lemma 3. If $\Delta$ and $\Delta^{*}$ are nonsingular, then $U^{*}\left(Z_{0}\right)=-U^{t}\left(Y_{0}\right)$.

Proof. We note that $Y_{0}$ and $Z_{0}$ exist and satisfy (1abd) and (2abd) respectively for $Q=R=I$. We find, by applying Lemma 1 , that

$$
\int_{a}^{b}\left[Z_{0}^{t} L\left(Y_{0} ; I\right)-L^{* t}\left(Z_{0} ; I\right) Y\right]=-U^{* t}\left(Z_{0}\right) V\left(Y_{0}\right)-V^{* t}\left(Z_{0}\right) U\left(Y_{0}\right) .
$$

The left side is 0 because of (1a) and (2a). The right side reduces to $-U^{* t}\left(Z_{0}\right)-U\left(Y_{0}\right)$ because of (1d) and (2d). Thus, $U^{*}\left(Z_{0}\right)=-U^{t}\left(Y_{0}\right)$.

Combining the results of Theorems 4 and 5 and Lemma 3 , we obtain the following:

THEOREM 6. If $\Delta$ and $\Delta^{*}$ are nonsingular, then there exists $a \neq 0$ for which system (1) is compatible if and only if there exists an $R \neq 0$ for which system (2) is compatible.

4. The Green's function. We obtain the Green's function by examining our solution to the nonhomogeneous system

(3) $L(Y ; Q)=F$ with side conditions (1bcd).

THEOREM 7. If $\Delta$ is nonsingular and, for every $Q$, system (1) is incompatible and $F$ is an integrable matrix, then there exists a $Q$ for which system (3) is compatible, with solution

$$
Y(x)=Y_{0}(x) Q+\int_{a}^{b} G_{0}(x, t) F(t) d t-\Phi(x) \Delta^{-1} V\left(\int_{a}^{b} G_{0} F\right),
$$

where

$$
Q=\left[U\left(Y_{0}\right)\right]^{-1}\left[U(\Phi) \Delta^{-1} V\left(\int_{a}^{b} G_{0} F\right)-U\left(\int_{a}^{b} G_{0} F\right)\right] .
$$


Proof. It is easily shown that the function $T(x)=\int_{a}^{b} G_{0}(x, t) F(t) d t$ satisfies $Y^{\prime}=P Y+F$. Hence, $Y$, given by $Y=\Phi C+J Q+T$, satisfies $L(Y ; Q)=F$. We need only to determine a suitable $C$ and $Q$. First, we apply $V$ to $Y$ to obtain $Q=\Delta C+V(J) Q+V(T)$. Solving for $C$ and replacing it in $Y$, we find that $Y=Y_{0} Q-\Phi \Delta^{-1} V(T)+T$. Now we apply $U$ to $Y$ to obtain

$$
U(Y)=U\left(Y_{0}\right) Q-U(\Phi) \Delta^{-1} V(T)+U(T)=0 .
$$

Since, for every $Q$, system (1) is incompatible, we know by Theorem 4 that $U\left(Y_{0}\right)$ is nonsingular so we may solve for $Q$. We have $Q$ $=\left[U\left(Y_{0}\right)\right]^{-1}\left[U(\Phi) \Delta^{-1} V(T)-U(T)\right]$ and $Y=Y_{0} Q+T-\Phi \Delta^{-1} V(T)$. Note that $Y-H^{t} Q=\left(Y_{0}-H^{t}\right) Q+T-\Phi \Delta^{-1} V(T)$, which is a.c. on $[a, b]$. Hence, $Y$ is a solution of (3).

To obtain a Green's function from the solution $Y$ of system (3) we write $Y$ in the form $\int_{a}^{b} G(x, t) F(t) d t$. This leads us to the following representation of the Green's function $G$, in which $U\left(Y_{0}\right)$ is denoted by $\Gamma$.

$$
\begin{aligned}
G(x, t)= & Y_{0}(x) \Gamma^{-1}\left[U(\Phi) \Delta^{-1} V\left(G_{0}\right)-U\left(G_{0}\right)\right] \\
& +G_{0}(x, t)-\Phi(x) \Delta^{-1} V\left(G_{0}\right)
\end{aligned}
$$

in which $U\left(G_{0}\right)$ and $V\left(G_{0}\right)$ are functions of $t$. Let

$$
\theta=\Gamma^{-1}\left[U(\Phi) \Delta^{-1} V\left(G_{0}\right)-U\left(G_{0}\right)\right] .
$$

Theorem 8. If $\Delta$ and $\Gamma$ are nonsingular and $G$ is defined by (4), then

(i) $L[G(x, \bar{t}) ; \theta]=0$ on $S_{h}$ except at $x=\bar{t}$,

(ii) $G(x, \bar{t})-H^{t}(x) \theta$ is a.c. on $[a, b]$ except at $x=\bar{t}$,

(iii) $U[G(x, \bar{t})]=0$,

(iv) $V[G(x, \bar{t})]=\theta$, and

(v) $G(t+, t)-G(t-, t)=I$, except when $t$ is at a discontinuity of $H$.

The proof consists of checking, using (4).

Theorems analogous to Theorems 7 and 8 hold for the adjoint system, of course.

A comparison of formula (4) and the formula for the Green's function in $[6$, p. 251] indicates the two are identical. In order to compare $G$ with Green's functions given in $[9$, p. 60] and $[3$, p. 564] we give an alternative formula for $G$, the tedious derivation of which relies on the formulas

$$
U\left(G_{0}\right)=\frac{1}{2}[-A \Phi(a)+B \Phi(b)] \Phi^{-1}(t)-J^{* t}(t)
$$


and

$$
\begin{aligned}
V\left(G_{0}\right)= & \frac{1}{2}[-C \Phi(a)+D \Phi(b)] \Phi^{-1}(t) \\
G(x, t)= & {\left[\Phi(x)-Y_{0}(x) \Gamma^{-1} U(\Phi)\right] \Delta^{-1} C \Phi(a) \Phi^{-1}(t) } \\
& +Y_{0}(x) \Gamma^{-1}\left[A \Phi(a)+\int_{a}^{t} d K \Phi\right] \Phi^{-1}(t) \quad \text { for } x>t \\
= & -\left[\Phi(x)-Y_{0}(x) \Gamma^{-1} U(\Phi)\right] \Delta^{-1} D \Phi(b) \Phi^{-1}(t) \\
& -Y_{0}(x) \Gamma^{-1}\left[B \Phi(b)+\int_{t}^{b} d K \Phi\right] \Phi^{-1}(t) \quad \text { for } t>x .
\end{aligned}
$$

In the case $H=0$, we have $Y_{0}=\Phi \Delta^{-1}$ so that $\Gamma^{-1}=\Delta[U(\Phi)]^{-1}$ and $Y_{0} \Gamma^{-1} U(\Phi)=\Phi$. It follows that (5) reduces to the form given in [9] and is essentially the same as that given in [3].

The relationship between $G$ and $G^{*}$, the Green's functions for systems (1) and (2) respectively, has been, in many previous papers, the motivation for the definition of the adjoint system. Here, we include it as a consequence of choice of adjoint systems.

THEOREM 9. If $\Delta$ and $\Delta^{*}$ are nonsingular and systems (1) and (2) are incompatible, then $G^{*}(x, t)=-G^{t}(t, x)$ for $x \neq t$.

Proof. Let $W$ and $X$ be any two continuous matrix functions, and let $Y$ and $Z$ be defined by $Y(x)=\int_{a}^{b} G(x, t) W(t) d t$ and $Z(t)$ $=\int_{a}^{b} G^{*}(t, x) X(x) d x$. Let $Q=V(Y)$ and $R=V^{*}(Z)$. Then $L(Y ; Q)=W$, $U(Y)=0, L^{*}(Z ; R)=-X$, and $U^{*}(Z)=0$. Applying Theorem 1 , we have $\int_{a}^{b}\left[Z^{t} W+X^{t} Y\right]=0$; i.e.,

$$
\int_{a}^{b} Z^{t}(t) W(t) d t+\int_{a}^{b} X^{t}(x) Y(x) d x=0 .
$$

It follows that

$$
0=\int_{a}^{b} \int_{a}^{b} X^{t}(x)\left[G^{* t}(t, x)+G(x, t)\right] W(t) d x d t
$$

Since our choice of $W$ and $X$ was arbitrary, we have, using the technique of Birkhoff and Langer [1, p. 61], $G^{* t}(t, x)+G(x, t)=0$ for $t \neq x$.

At this point it should be noted that several forms of interface conditions have appeared in the literature, including the following: 
(a) $Y\left(t_{i}+\right)=A_{i} Y\left(t_{i}-\right), \quad i=1,2, \cdots, m \quad$ in [8] and [10] (b) $A_{i} Y\left(t_{i}+\right)+B_{i} Y\left(t_{i}-\right)=C_{i}, \quad i=1, \cdots, m$ in [4] and

(c) $\sum_{0}^{m}\left[A_{i j} Y\left(t_{j}+\right)+B_{i j} Y\left(t_{j}-\right)\right]=0, \quad i=1, \cdots, k \quad$ in [5] (d) $Y(t+)-Y(t-)=J_{t}$ in [2], [3], and this paper.

None includes all others as special cases, though (a) is obviously included in (b) as is (d) in the case of only finitely many points. A natural question arises concerning the form of the adjoint of a system similar to (1) with condition (1b) replaced by something like $\sum_{\alpha}\left[A_{i \alpha} Y\left(t_{\alpha}+\right)+B_{i \alpha} Y\left(t_{\alpha}-\right)\right]=C_{i}, i=1,2, \cdots, k$, in which $\alpha$ ranges over a set which could be infinite.

\section{BIBLIOGRAPHY}

1. G. D. Birkhoff and R. E. Langer, The boundary problems and development associated with a system of ordinary linear differential equations of the first order, Proc. Amer. Acad. Arts Sci. 58 (1923), 51-128.

2. R. N. Bryan, An adjoint system for a nonhomogeneous linear differential system (to appear).

3. R. H. Cole, General boundary conditions for an ordinary differential system, Trans. Amer. Math. Soc. 111 (1964), 521-550.

4. R. Conti, On ordinary differential equations with interface conditions, J. Differential Equations 4 (1968), 4-11.

5. A. M. Krall, Differential operators and their adjoints under integral and multiple point boundary conditions, J. Differential Equations 4 (1968), 327-336.

6. - , Nonhomogeneous differential operators, Michigan Math. J. 12 (1965), $247-255$.

7. T. J. Pignani, and W. M. Whyburn, Differential systems with interface and general boundary conditions, J. of the Elisha Mitchell Scientific Soc. 72 (May 1956), $1-14$.

8. F. W. Stallard, Functions of bounded variation as solutions of differential systems, Proc. Amer. Math. Soc. 13 (1962), 366-373.

9. W. M. Whyburn, Differential systems with general boundary conditions, Seminar Reports in Math, Univ. of Calif., Pub. in Math. 2 (1944), 45-61.

10. A. Zettl, Adjoint and self-adjoint boundary value problems with interface conditions, Math. Res. Center, Tech. Report No. 827, 1967.

IthaCA COLlege 